\title{
Ictal and interictal MEG in pediatric patients with tuberous sclerosis and drug resistant epilepsy
}

Koptelova, A.

2018-02

Koptelova , A, Bikmullina , R, Medvedovsky , M , Novikova , S, Golovteev , A, Grinenko , O, Korsakova , M , Kozlova , A, Arkhipova , N, Vorobyev, A, Melikyan , A, Paetau , R, Stroganova , T \& Metsähonkala , L 2018 , ' Ictal and interictal MEG in pediatric patients with tuberous sclerosis and drug resistant epilepsy ' , Epilepsy Research , vol. 140 , pp. 162-165 . https://doi.org/10.1016

http://hdl.handle.net/10138/301306

https://doi.org/10.1016/j.eplepsyres.2017.12.014

publishedVersion

Downloaded from Helda, University of Helsinki institutional repository.

This is an electronic reprint of the original article.

This reprint may differ from the original in pagination and typographic detail.

Please cite the original version. 


\title{
Ictal and interictal MEG in pediatric patients with tuberous sclerosis and drug resistant epilepsy
}

\author{
A. Koptelova ${ }^{\mathrm{a}, *, 1}$, R. Bikmullina ${ }^{\mathrm{b}, 1}$, M. Medvedovsky ${ }^{\mathrm{c}}, \mathrm{S}$. Novikova ${ }^{\mathrm{a}}$, A. Golovteev ${ }^{\mathrm{e}, \mathrm{g}}$, \\ O. Grinenko ${ }^{e}$, M. Korsakova ${ }^{e}$, A. Kozlova ${ }^{e}$, N. Arkhipova ${ }^{\mathrm{e}}$, A. Vorobyev $^{\mathrm{e}}$, A. Melikyan $^{\mathrm{e}}$, \\ R. Paetau ${ }^{\mathrm{d}, \mathrm{f}}$, T. Stroganova ${ }^{\mathrm{a}, \mathrm{h}}$, L. Metsähonkala ${ }^{\mathrm{d}}$ \\ ${ }^{\text {a }}$ Center for Neurocognitive Research (MEG center), Moscow State University of Psychology and Education, Shelepikhinskaya Naberezhnaya 2a, 123290 Moscow, Russia

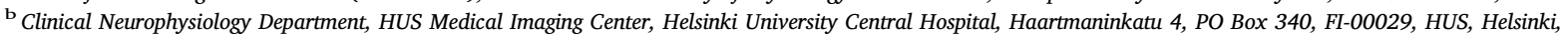 \\ Finland \\ ' Sagol Brain Institute, Tel-Aviv Sourasky Medical Center, Weizmann St 6, 6423906 Tel-Aviv, Israel \\ d Children's Hospital, Pediatric Neurology, University of Helsinki and Helsinki University Hospital, Stenbäckinkatu 9, PO BOX 100, FI-00029, HUS Helsinki, Finland \\ ${ }^{\mathrm{e}}$ N.N. Burdenko National Medical Research Center of Neurosurgery of the Ministry of Health of the Russian Federation (N.N. Burdenko NMRCN), 4th Tverskaya- \\ Yamskaya street 16, 125047 Moscow, Russia \\ ${ }^{\mathrm{f}}$ Clinical Neurosciences, Biomag laboratory, University of Helsinki and Helsinki University Hospital, Haartmanninkatu 4, PO BOX 340 , FI-00029 HUS Helsinki, Finland \\ ${ }^{\mathrm{g}}$ Epilepsy Center, Moscow, Visokovoltnyi proezd 1/3, 127556 Moscow, Russia \\ h Autism Research Laboratory, Moscow State University of Psychology and Education, Shelepikhinskaya Naberezhnaya 2a, 123290 Moscow, Russia
}

\section{A R T I C L E I N F O}

\section{Keywords:}

Epilepsy surgery

Tuberous sclerosis

Ictal and interictal MEG

\begin{abstract}
A B S T R A C T
Purpose: Drug resistant epilepsy (DRE) is common in patients with tuberous sclerosis (TS). Interictal MEG has been shown as a valuable instrument in the presurgical workup. The goal of our study was to evaluate the role of ictal MEG in epileptogenic tuber selection, especially in patients with multiple irritative zones.

Methods: The clinical and MEG data of 23 patients with TS and DRE from two medical/research centers were reviewed. Seven pediatric patients, who had seizures during MEG recording and underwent resection or disconnection surgery, were included into the study. Cortical sources of ictal and interictal epileptiform MEG discharges were compared with epileptogenic zone location in six patients with favorable surgery outcome. Results: In patients who improved substantially after surgery all resected and several other tubers demonstrated epileptiform activity on interictal MEG. Ictal MEG provided crucial information about lobar location of the seizure onset zone (SOZ) in two cases, and in the other four it confirmed the SOZ location derived from the interictal data. In one case, ictal MEG findings were unreliable. In one patient, who did not benefit from surgical treatment, the resected tubers did not overlap with interictal and ictal MEG sources.

Conclusion: The combination of interictal and ictal MEG is a valuable tool for identification of the epileptogenic tuber/tubers in presurgical work-up in patients with TS.
\end{abstract}

\section{Introduction}

Epilepsy with tuberous sclerosis (TS) is often drug resistant, and some patients benefit from surgical intervention. Due to the multifocal nature of TS, it is often difficult to decide, which tuber (or tubers) should be removed to achieve freedom of seizures. Previous studies of patients with TS and drug resistant epilepsy (DRE) have demonstrated interictal MEG to be useful for identifying a tuber generating epileptiform activity (Iida et al., 2005; Jansen et al., 2006; Wu et al., 2006; Kamimura et al., 2006). However, several tubers can produce interictal epileptiform discharges, whereas only some of them are responsible for seizure initiation (Wu et al., 2006). Here we aimed to investigate whether ictal MEG can be more specific than interictal MEG in selecting epileptogenic tubers from several interictally active ones, and to reveal the added value of ictal MEG in the presurgical workup of TS patients with multiple electrographically active tubers.

\section{Methods}

This study was approved by the ethical committees of Moscow State

\footnotetext{
Abbreviations: DRE, drug resistant epilepsy; TS, tuberous sclerosis; EZ, epileptogenic zone; SOZ, seizure onset zone; SDC, supplementary digital content

* Corresponding author.

E-mail address: koptelova.am@gmail.com (A. Koptelova).

${ }^{1}$ The authors contributed equally to the article.
} 
University of Psychology \& Education (MSUPE), Moscow, Russia and E7 of Helsinki University Hospital (HUH), Helsinki, Finland.

This study includes the data of TS patients from the epilepsy surgery programs of N.N. Burdenko NMRCN, Moscow, Russia (2010-2017) and of HUH (1995-2017). The inclusion criteria were: a presence of TS with DRE, interictal and ictal MEG and resection or disconnection surgery. Twenty-three TS patients were referred to MEG, and seizures of 13 patients were recorded. Seven of them underwent resection or disconnection surgery.

The following information was collected from the medical records: age, gender, seizure semiology, ictal and interictal data from video-EEG and MEG, MRI, resection site, post-surgery follow-up duration and surgery outcome. Informed consent was obtained from all patients or their caregivers.

MEG was recorded using Elekta-Neuromag 306-sensor MEG device (102 magnetometers, 204 planar gradiometers) in MEG center of MSUPE and in BioMag laboratory of HUH. The data were analyzed using the manufacturer's software. Spatio-temporal source separation (Taulu and Simola, 2006) and the head position were monitored continuously. Epileptiform signals were identified by MEG experts (TS, AK, RP and MM); the sources were localized using manual sequential multidipole fit (Salmelin, 2010). The sources were co-registered with patients' MRI.

The ictal events were defined according to ILAE recommendations and were related to an onset of the clinical seizure manifestations (Fisher et al., 2005). The first MEG signal change temporally related to the clinical seizure onset was used for ictal source localization. In 6 patients, the MEG signal changed before the clinical seizure onset (preictal MEG signal); in one patient (case 6) such change was identified after appearance of clinical signs, and in this case the ictal sources were localized from the first subtle epileptic spasms that did not cause a movement artifact.

Interictal MEG source clusters were defined as six or more sources with $\leq 1 \mathrm{~cm}$ between adjacent sources (Iida at al. 2005). Among them, we separated major interictal clusters that contained either the sources of continuous local epileptiform transients rarely interrupted by physiological activity or the initial sources of prolonged rhythmic discharges/electrographic seizures.

\section{Results}

The data of seven patients (six from MSUPE and one from HUH) are presented in Table 1. The case reports can be found in Supplemental Digital Content (SDC).

\subsection{Surgery outcome}

Post-surgery follow-up varied from one to four years. Four patients achieved seizure freedom (Engel I). In two patients, only rare seizures remained (Engel II). Thus, the results indicated that in six cases the epileptogenic tubers were likely to have been removed or disconnected. In one patient, surgery did not change the seizure frequency or severity indicating a failure to identify the epileptogenic zone (Engel IVB).

\subsection{Ictal vs interictal $M E G$}

In all six patients with favorable surgery outcome, the resected areas contained major MEG source clusters (see SDC). Considering that these patients substantially improved after surgery, we expect that the resected major clusters corresponded to epileptogenic zone (EZ). However, in all cases, interictal activity was also present outside the resected areas, including discharges generated by one of the major clusters (patients 2 and 6). This prevented an unambiguous EZ identification based solely on their interictal MEG. Ictal MEG provided a correct localization of the EZ at the lobar level in five $(1,2,4,5,6)$ patients. In two cases (patients 2 and 6) ictal MEG findings were crucial, because they allowed selecting EZ among several major clusters/irritative zones. In patients 1, 4 and 5 ictal MEG confirmed localization of EZ derived from the interictal data, which reported a single major cluster. The ictally localized EZ in all 5 cases overlapped with one of the major interictal clusters. However, at the sub-lobar level, ictal MEG was not always accurate enough (patient 1 ). In patient 3, ictal MEG findings were misleading: the epileptogenic tuber was located in the other hemisphere and its surgical removal led to favorable outcome. The inaccurately identified EZ by ictal MEG (i) did not coincide with the interictal major cluster, which was located in the vicinity of the resected tuber and (ii) the ictal MEG sources were scattered across the whole hemisphere. Due to the widespread distribution of ictal signal, the MEG interpreters reported this ictal MEG finding as unreliable.

In the patient 7 , who did not benefit from surgical treatment, the area of resected tubers overlapped neither with the major interictal MEG cluster nor with the ictal MEG sources (Table 1 and SDC).

\subsection{Ictal EEG vs ictal MEG}

In four out of six patients with favorable surgery outcomes, ictal EEG neither lateralized nor localized the EZ (patients 1, 2, 3 and 4). In three of these cases (patients 1, 2 and 4), ictal MEG provided EZ localization to the correct lobe, and in one (patient 3) ictal MEG was mislateralizing. In patients 5 and 6 ictal EEG and ictal MEG were concordant on one tuber, but in patient 6 MEG suggested simultaneous ictal onset activity in an additional tuber. In patient 7 , the ictal EEG and ictal MEG results were discordant. Ictal EEG suggested the epileptogenic tuber, whose resection did not lead to any changes in patient's seizures.

\section{Discussion}

We reported interictal and ictal MEG findings in seven TS patients, who underwent resection or disconnection surgery, six of them with favorable outcome and one with surgery failure. Previous MEG studies in TS patients with DRE were mostly limited to interictal recordings (Iida et al., 2005; Jansen et al., 2006; Wu et al., 2006; Kamimura et al., 2006; Medvedovsky et al., 2012). In our study, in six TS patients with successful surgery, interictal MEG did not miss any tuber or brain region whose removal led to favorable surgery outcome in accordance with the previous results (Jansen et al., 2006; Wu et al., 2006; Kamimura et al., 2006). Although in each individual study, including ours, the sample size of TS patients with surgically verified EZ localization was small (up to 6 patients), the concordance of the results implies that interictally "MEG-silent" tubers are not usually epileptogenic.

Also, consistently with the previous reports (Jansen et al., 2006; Wu et al., 2006; Kamimura et al., 2006), a single major interictal cluster accurately predicted localization of the EZ in four our patients. However, if several major interictal clusters were demonstrated, which was the case in another two patients, the interictal MEG was unable to determine the EZ. In these two patients, ictal MEG correctly identified the epileptogenic tuber/tubers at the lobar level among several irritative zones. Thus, the TS patients with multifocal epileptiform discharges in their interictal MEG/EEG are expected to benefit most from a combination of interictal and ictal MEG during their pre-surgical evaluation. However, localization accuracy of ictal MEG can be limited if the signal-to-noise ratio at the beginning of ictal activity is low and the activity propagation from seizure onset zone is fast. The clear indications of ictal MEG failure in one of our patients were the widespread cortical sources of pre-ictal MEG pattern and discordance between ictal MEG finding and location of the single major interictal cluster. Reliability criteria for ictal MEG are currently missing and should be elaborated in future. Another issue is the selection of optimal source localization model for fast oscillating ictal-onset signal. The use of sLORETA and SAM(G2) beamformer for ictal onset MEG source localization has been reported (Fujiwara et al., 2012), but here again, further studies are needed. 


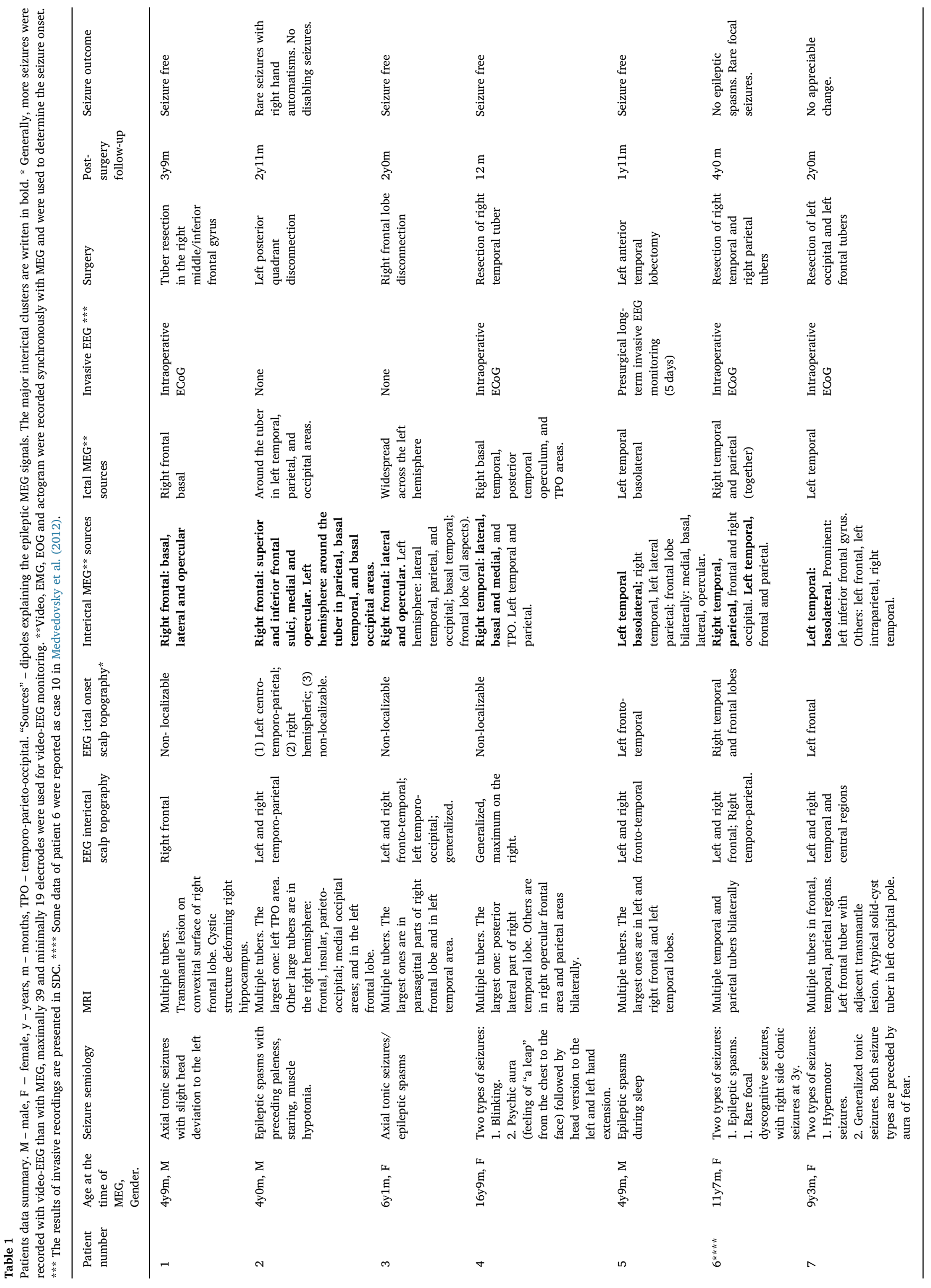


The limitations of the current study are the small sample size and relatively short post-surgery follow-up. Therefore, despite the encouraging evidence on a usefulness of a combination of interictal and ictal MEG for epileptogenic tuber identification, more studies are needed to enhance the certainty of this conclusion.

\section{Conclusions}

1. In patients with TS the epileptogenic zone(s) most likely coincides with some of interictally active tuber(s) producing epileptiform MEG activity.

2. Ictal MEG as compared to interictal MEG may provide unique information for identifying the epileptogenic zone in patients with multiple interictally active tubers, and for such TS patients ictal MEG should be recommended.

3. Clear reliability criteria for ictal MEG analysis should be elaborated

4. The combination of interictal and ictal MEG is a valuable tool in presurgical work-up in patients with TS.

\section{Acknowledgments}

The study was supported by the core funding of MEG Center MSUPE and by the Finnish governmental subsidiary TYH2015309.

\section{Appendix A. Supplementary data}

Supplementary data associated with this article can be found, in the online version, at https://doi.org/10.1016/j.eplepsyres.2017.12.014.

\section{References}

Fisher, R.S., Boas, W.V.E., Blume, W., Elger, C., Genton, P., Lee, P., Engel, J., 2005 Epileptic seizures and epilepsy: definitions proposed by the international league against epilepsy (ILAE) and the international bureau for epilepsy (IBE). Epilepsia 46 (4), 470-472.

Fujiwara, H., Greiner, H.M., Hemasilpin, N., Lee, K.H., Holland-Bouley, K., Arthur, T., Morita, D., Jain, S.V., Mangano, F.T., deGrauw, T., Rose, D.F., 2012. Ictal MEG onset source localization compared to intracranial EEG and outcome: improved epilepsy presurgical evaluation in pediatrics. Epilepsy Res. 99 (3), 214-224.

Iida, K., Otsubo, H., Mohamed, I.S., Okuda, C., Ochi, A., Weiss, S.K., Chuang, S.H., Snead, O.C., 2005. Characterizing magnetoencephalographic spike sources in children with tuberous sclerosis complex. Epilepsia 46 (9), 1510-1517.

Jansen, F.E., Huiskamp, G., Huffelen, A.C., Bourez-Swart, M., Boere, E., Gebbink, T., Vincken, K.L., Nieuwenhuizen, O., 2006. Identification of the epileptogenic tuber in patients with tuberous sclerosis: a comparison of high-resolution EEG and MEG. Epilepsia 47 (1), 108-114.

Kamimura, T., Tohyama, J., Oishi, M., Akasaka, N., Kanazawa, O., Sasagawa, M., Kato, M., Ohno, K., Masuda, H., Kameyama, S., Uchiyama, M., 2006.

Magnetoencephalography in patients with tuberous sclerosis and localization-related epilepsy. Epilepsia 47 (6), 991-997.

Medvedovsky, M., Taulu, S., Gaily, E., Metsähonkala, E.L., Mäkelä, J.P., Ekstein, D., Kipervasser, S., Neufeld, M., Kramer, U., Blomstedt, G., Fried, I., Karppinen, A., Veshchev, I., Roivainen Ben-Zeev, B., Goldberg-Stern, H., Wilenius, J., Paetau, R, 2012. Sensitivity and specificity of seizure-onset zone estimation by ictal magnetoencephalography. Epilepsia 53 (9), 8207.

Salmelin, R., 2010. Multi-dipole modelling in MEG. In: Hansen, P.C., KringElbach, M.L., Salmelin, R. (Eds.), MEG: An Introduction to Methods. Oxford University Press, pp. $124-155$.

Taulu, S., Simola, J., 2006. Spatiotemporal signal space separation method for rejecting nearby interference in MEG measurements. Phys. Med. Biol. 51 (7), 1759.

Wu, J.Y., Sutherling, W.W., Koh, S., Salamon, N., Jonas, R., Yudovin, S., Sankar, R., Shields, W.D., Mathern, G.W., 2006. Magnetic source imaging localizes epileptogenic zone in children with tuberous sclerosis complex. Neurology 66 (8), 1270-1272. 